\title{
Porphyrias - diagnostic challenge in Emergency room
}

\author{
MILA LOVRIĆ \\ Department of Laboratory Diagnostics, University Hospital Centre Zagreb, Zagreb, Croatia
}

Corresponding author:

Mila Lovrić

Department of Laboratory Diagnostics

University Hospital Centre Zagreb, Zagreb, Croatia

Kišpatićeva 12, 10000 Zagreb

Phone: 38512367 249; fax: 38512367395

E-mail:mila.lovric@kbc-zagreb.hr

\section{ABSTRACT}

Porphyrias form a heterogeneous group of disorders of haem biosynthesis. They are often missed or wrongly diagnosed in the emergency department. Acute porphyrias present most commonly as abdominal pain or as neurological or atypical psychiatric symptoms. The variety of clinical features may delay diagnosis, and unrecognized disease is potentially life-threatening. The frequency and severity of attacks vary widely. In some people, this disease remains latent throughout life, even in the presence of precipitating factors. Other people experience frequent and sometimes life-threatening attacks even in the apparent absence of exogenous precipitating factors.

Laboratory diagnosis of acute porphyria includes porphyrin precursors delta aminolevulic acid and porphobilinogen. Patients presenting with acute crises often have several-fold increases in PBG above the reference interval, usually $>10$ times the upper reference limit. Hyponatremia and rabdomyolysis are relatively common manifestation accompanying acute attack.

Key words: porphyria, laboratory diagnostic, emergency room

\section{INTRODUCTION}

Porphyrias are a group of metabolic diseases caused by defect in one of the seven enzymes involved in the haem biosynthesis pathway (figure 1). They form a heterogeneous group of disorders of haem biosynthesis, and they are often missed or wrongly diagnosed. Partial deficiency of one of the seven enzymes in the pathway causes characteristic clinical and biochemical features. These disorders are due to a specific alteration in the pattern of accumulation of porphyrin and porphyrin precursors. Each type of porphyria is defined by a unique pattern of accumulation and excretion of haem precursors, as well as a reduction in the relevant enzyme activity. A correct interpretation of the appropriate biochemical investigation is essential to accurately diagnose and manage porphyrias, as clinical features alone are not sufficiently specific to either confirm the diagnosis or to distinguish between the various forms. Porphyrias are classified on the clinical basis into acute porphyrias (characterized by neuropsychiatric symptoms) or cutaneous porphyrias (characterized by photosensitivity to sunlight). Porphyrias are also classified as erythropoietic porphyria or hepatic porphyrias, depending on whether the genetic defect mainly affects the erythroid or the hepatic haem biosynthesis. There are four acute hepatic porphyrias characterized by neurologic and potentially life-threatening acute attacks: acute intermittent porphyria (AIP), variegate porphyria (VP), hereditary coproporphyria (HCP) and 5-aminolevulinic dehydratase deficiency porphyria (ALADdeficiency porphyria). The symptoms of AIP and ALAD-deficiency porphyria are essentially neurological, whereas VP and HCP are mixed forms which may present with either neurovisceral manifestations or cutaneous photosensitivity or both. AIP is the most commonly encountered acute porphyria worldwide and has a higher clinical penetrance compared with AP and HCP. ALAD deficiency porphyria is extremely rare and inherited in recessive form. Acute hepatic porphyria is characterized by acute neuropsychiatric and potentially life-threatening attacks precipitated by endogenous or environmental factors such as reproductive hormones, fasting, stress, infection or porphyrinogenic drugs and alcohol - that is, factors increasing the requirements for hepatic haem biosynthesis. The onset of symptoms almost never occur before puberty, and women of fertile age are more affected than men, implying special susceptibility to reproductive hormones. AIP may serve as a general model for the clinical management of acute porphyria crises, since treatment, diagnostic and preventive measures are similar in all acute porphyrias.

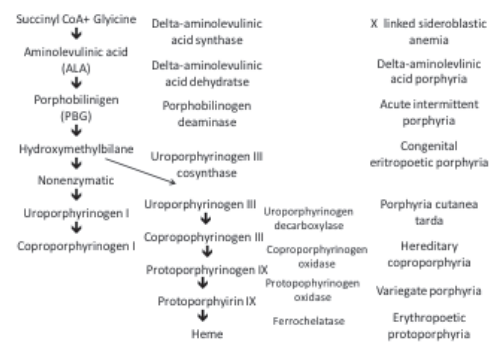

Figure 1. Haem synthesis: metabolites, enzymes and disorders associated with enzyme deficiencies.

\section{CLINICAL MANIFESTATION OF ACUTE INTERMITTENT PORPHYRIA ATTACK}

Acute attacks are often triggered by exposure to exogenous participating factors, including a wide range of commonly prescribed drugs. The frequency and severity of attacks vary widely and are rare before puberty. They are most common in people in their thirties and are four to five times more common in females than in males, with a peak age of presentation in the early 
30s.

In some people the disease remains latent throughout life, even in the presence of precipitating factors. Other people experience frequent and sometimes life-threatening attacks even in the apparent absence of exogenous precipitating factors.

The manifestation of acute porphyria attacks are mainly neurological and accompanied by metabolic manifestation. The variety of clinical features may delay diagnosis, and unrecognized the disease is potentially life-threatening. The most common symptom is severe abdominal pain without peritoneal signs, present in over $90 \%$ of patients. Hypertension and tachycardia are generally present. Hyponatremia may take place due to sodium loss, overhydration or hypothalamic involvement. Seizures can be a consequence of hyponatremia or hypomagnesemia, or be secondary to CNS involvement. Other general symptoms often accompanying acute attacks are tiredness, restlessness and headaches. Patients must be evaluated clinically to exclude other causative clinical conditions.

\section{LABORATORY DIAGNOSIS OF ACUTE INTERMITTENT PORPHYRIA}

The porphyrin precursors delta aminolevulic acid (ALA) and PBG are invariably increased when neuropsychiatric illness is caused by AIP. Urine is usually coloured red-brown on standing due to PBG oxidation into porphobilin. Urine discoloration is a trait described in a large number of case reports as a feature leading to AIP diagnosis. The expected colour-based diagnosis may be absent in patients with ongoing fluid administration or increased urinary frequency.

Laboratory qualitative screening test to confirm the presence of PBG should be available in an acute clinical laboratory. False-negative and false-positive results are not uncommon, and if a result is uncertain the semiquantitative test should be available. Screening test should be followed by quantitative analysis of ALA and PBG. Urine ALA and PBG are measured by ion exchange chromatography and plasma determined by the HPLC-mass spectrometry method. The ratio may be important in the AIP surveillance program, as an increasing ratio may indicate impaired renal function. The ratio of PBG and ALA in urine ( $\mathrm{mmol} /$ mol creatinine) or plasma $(\mu \mathrm{mol} / \mathrm{L})$ is approximately 0.3 in healthy individuals and in latent AIP carriers. In AIP patients with chronic high excretion, the ratio is approximately 2.0.

Only a few carriers present high concentrations of ALA and PBG secondary to 50\% a PBGD activity. Some display an asymptomatic clinical pattern, while others may present with acute or sub-acute chronic symptoms. Patients presenting with acute crises often have several-fold increases in PBG above the reference interval, usually > 10 times the upper reference limit.

AIP diagnosis must always, if possible, be followed by gene analysis.

Most hospital wards have packages of analyses applied in care for patients with porphyrias; general laboratory tests should include full blood status, liver and kidney function test and electrolytes. Recent studies have shown that the following tests to assess hepatic inflammation may be useful: C-reactive protein, clotting function and serum ferritin should be monitored during treatment.

\section{HYPONATREMIA}

Hyponatremia is a relatively common metabolic manifestation accompanying acute attack. It is usually mild and easily corrected. It is proposed to be secondary to excessive loss due to vomiting, diarrhoea, sweating or increased renal loss due to osmotic diuresis after glucose infusion and iatrogenic fluid substitute without electrolyte replacement. Hyponatremia can also be found on admission of acute attack patients without the proposed mechanisms, and antidiuretic hormone has also been suggested as a possible factor. Hyponatremia in AIP may cause seizures and encephalopathy, while severe hyponatremia may lead to brain edema. Hyponatremia is found in a number of disorders of CNS, and thus AIP should be kept in mind as a differential diagnosis. In unknown carriers, an overlapping of symptoms may delay AIP diagnosis.

\section{RHABDOMYOLYSIS}

Rhabdomyolysis in AIP attacks is accompanied by important muscle waste and myoglobinuria, and is probably more common than the few reported cases in literature would suggest. The condition presents with important elevation of creatinine kinase (CK) levels, as well as AST, often concurrent with hyponatremia and other symptoms such as abdominal pain, vomiting and muscle pain. The cause of rabdomyolysis in AIP is not clear

\section{REFERENCES}

1. Harper P, Sardh E. Management of acute intermittent porphyria. Expert Opinion on Orphan drugs 2014:2;349-362.

2. Minder EI, Schneider-Yin X. Porphyrins, porphobilinogen and $\delta$-aminolevulic acid. U: Blau N, Duran M, Gibson KM. Laboratory guide to the methods in biochemical genetics. Berlin-Heidelberg: Springer Verlag, 2008;751-80.

3. Deacon A, Whatley SD, Elder GH. Porphyrins and Disorders of Porphyrin Metabolism. U Burtis CA, Ashwood ER, DE Bruns: Tietz textbook of clinical chemistry and molecular diagnostics. St Louis, SAD; Elsevier Saunders, 2006; 527-38.

4. Sassa S. Modern diagnosis and management of the porphyrias. British Journal of Haematology 2006: 135; 281-92.

5. Thadani H, Deacon A, Peters T. Diagnosis and management of porphyria, BMJ 2000:320;1647-51.

6. Foran SE, Abel G. Guide to Porphyrias: A Historical and Clinical Perspective, Am J Clin Pathol 2003: 119;87-93.

7. Arsanad AK, Villanger JH, Stole E, Deybach JC, Marsden J, To-Figueras J, Badminton M, Elder GH, Sandberg S. European Specialst Porphyria Laboratory: Diagnostic Strategies, Analytical Quality, Clinical Interpretation, and Reporting As Assessed by External Quality Assurance Program, Clin Chem 2011: 57;1514-23. 